\title{
Pressure Effects in ortho-Positronium Annihilation
}

\author{
T. Goworek, J. WaWryszczuk and R. Zaleski
}

Institute of Physics, Maria Curie Skłodowska University pl. Marii Curie-Skłodowskiej 1, 20-031 Lublin, Poland

A short review of experiments concerning the influence of high pressure on positron annihilation in molecular solids is given. To this kind of experiments belong e.g. pressure inhibition of positronium formation, pressure induced phase transitions, and free volume swelling in the media intercalated by high pressure gas.

PACS numbers: 36.10.Dr, 78.70.Bj

\section{Introduction}

In the literature on positron annihilation lifetime spectroscopy (PALS) numerous papers are devoted to the temperature dependence of annihilation parameters: lifetime and relative intensity of various modes of decay. The number of studies on pressure dependence of the same parameters is in great contrast to the previously mentioned: the number is very low. The pressure studies certainly deserve more attention. Using quite moderate pressures one can observe many interesting effects. In this paper we want to describe some of them. The data presented below were obtained in positron laboratory at Marie Curie-Skłodowska University in Lublin. All the spectra were measured on standard fast-slow spectrometers $\left(\mathrm{BaF}_{2}\right.$ crystals, dynode output) and processed using the LT programme [1].

\section{Disappearance of energy level for a particle in the potential well}

Positronium atom $(\mathrm{Ps})$ is trapped in the volumes of low electron density in the solid due to negative Ps work function. Free volume constitutes a potential well whose depth is equal to that work function [2]. The zero-point energy of a particle confined in the potential well is inversely proportional to the square of the well size. If the free volume constitutes rectangular potential well with spherical symmetry, the zero point energy becomes equal to the well depth $V$ at the radius

$$
R_{\min }=h / 4(2 m V)^{1 / 2}
$$


( $h, m$ are the Planck constant and particle mass, respectively). At well radii smaller than $R_{\min }$ there are no levels for the particle in the well. For positronium the lack of level means no possibility of trapping and thus, there is no long-lived component characteristic of ortho-Ps (o-Ps) in the PALS spectrum.

In some molecular crystals, e.g. of aromatic compounds, the free volumes are thermally produced; for naphthalene, biphenyl, and chrysene the activation enthalpy of these Ps centres is about $0.2 \mathrm{eV}$. One can suppose that these centres, produced due to the lattice dynamics, are prone to the action of pressure their sizes should diminish relatively easy with the increase in pressure and after reduction below $R_{\min }$ the $o$-Ps component should disappear. The elimination of $o$-Ps by pressure was demonstrated for the first time in the case of biphenyl [3]. In Fig. 1 we show the pressure dependence of $o$-Ps intensity $I_{3}$ and lifetime $\tau_{3}$ for naphthalene [4]. The sandwich of zone refined polycrystalline naphthalene and positron source ${ }^{22} \mathrm{Na}$ was placed in a steel cylinder; the sandwich filled the whole volume beneath the piston producing the pressure. It is seen in Fig. 1 that the lifetime $\tau_{3}$, ruled by the free volume size, decreases continuously, but the intensity $I_{3}$, initially weakly dependent on the pressure, at about $100 \mathrm{MPa}$ decreases suddenly till its total disappearance.

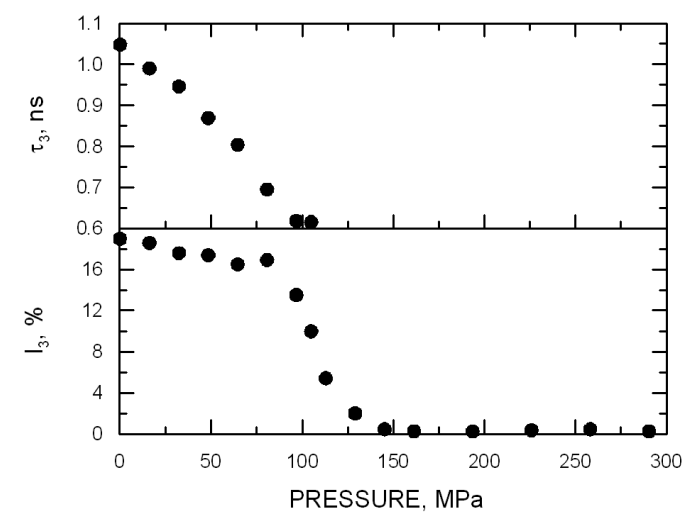

Fig. 1. The long-lived component lifetime $\tau_{3}$ (top) and intensity $I_{3}$ (bottom) in naphthalene as a function of pressure [4].

Free volumes (voids) in molecular crystals can be created by introduction to the structure strange molecules crystallizing together with the main component, but smaller in size. This way small empty volumes are produced in the vicinity of the dopant; the size of such a free volume is smaller than that expected for a vacancy. $p$-terphenyl with an admixture of anthracene (in our case $0.6 \%$ of admixture) can serve as an example of doped crystal, containing the centres of Ps formation. Figure 2 shows the temperature dependence of $o$-Ps lifetime and intensity, while Fig. 3 shows the pressure dependence. 


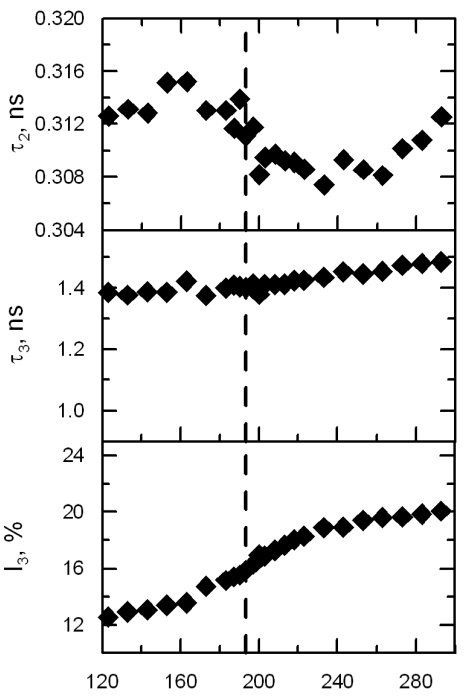

Fig. 2 TEMPERATURE, $\mathrm{K}$

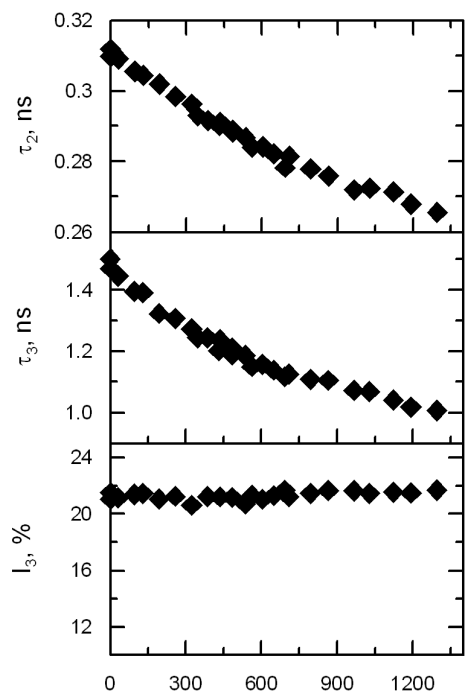

Fig. 3 PRESSURE, MPa

Fig. 2. Positron lifetime parameters in $p$-terphenyl with $0.6 \%$ admixture of anthracene as a function of temperature. From top to bottom: free annihilation lifetime $\tau_{2}$, ortho-Ps lifetime $\tau_{3}$, ortho-Ps intensity $I_{3}$. Dashed vertical line indicates the phase transition point.

Fig. 3. Positron lifetime parameters in $p$-terphenyl with $0.6 \%$ admixture of anthracene as a function of pressure (room temperature). From top to bottom: free annihilation lifetime $\tau_{2}$, ortho-Ps lifetime $\tau_{3}$, ortho-Ps intensity $I_{3}$.

It is known that there is a solid-solid phase transition in this crystal at $T_{\mathrm{tr}}=193 \mathrm{~K}$, very faintly visible in the form of minute change of free annihilation lifetime $\tau_{2}$. In the pressure studies this phase transition cannot be observed at room temperature, because $\mathrm{d} T_{\mathrm{tr}} / \mathrm{d} p$ is negative and with the increase in pressure it shifts down; at $550 \mathrm{MPa}$ the transition point falls to $0 \mathrm{~K}$ [5]. From Figs. 2 and 3 one can see that the temperature and pressure dependences of annihilation parameters are dissimilar. In spite of application of very large pressures the intensity $I_{3}$ is insensitive to pressure, while it decreases substantially when the temperature is lowered. On the contrary, the lifetimes strongly depend on pressure, while they are almost constant in a broad range of temperatures. At $1300 \mathrm{MPa}$ the lifetime $\tau_{3}$, although shortened by $32 \%$ comparing to $p=0$, is still much longer than it was observed in the case of naphthalene. Free volume near the anthracene molecule located between the longer $p$-terphenyl neighbours is hard to compress to the values below $R_{\min }$.

\section{Pressure induced phase transitions}

When $\mathrm{d} T_{\mathrm{tr}} / \mathrm{d} p$ is positive, the increase in pressure is equivalent to temperature lowering. Such a situation appears in long-chain alkanes [6]. At low temper- 
atures the alkane crystals are in the so-called rigid phase, but $5-10 \mathrm{~K}$ below the melting point they undergo the transition to waxy (rotator) phase. In the rotator phase containing great number of nonplanar conformers [7] the $o$-Ps lifetime is almost twice of that in the rigid phase, thus it is easily observed by PALS. Figure 4 shows the result of $o$-Ps lifetime measurement in $n$-nonadecane, $\mathrm{C}_{19} \mathrm{H}_{40}$ at $302 \mathrm{~K}$ ( $3 \mathrm{~K}$ below the melting point). The lifetime decreases very slowly with pressure and at $32 \mathrm{MPa}$ the sample transforms to the rigid phase reducing $\tau_{3}$ to $1.6 \mathrm{~ns}$.

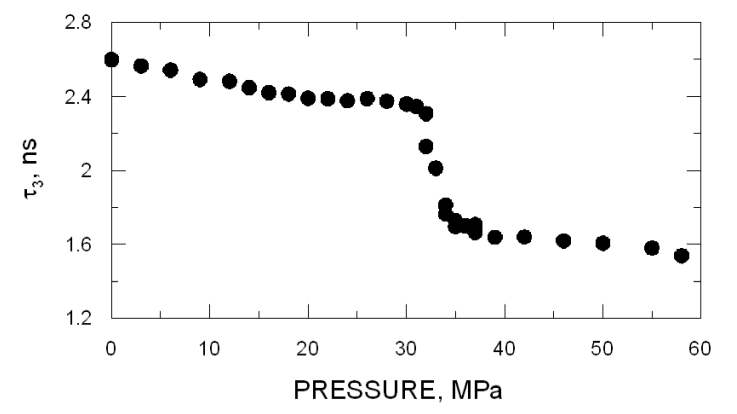

Fig. 4. ortho-Ps lifetime in $n$-nonadecane as a function of pressure. Temperature $302 \mathrm{~K}$.

Further increase in pressure gives little effect in annihilation parameters. The lifetime above $\approx 600 \mathrm{MPa}$ is almost constant. It is known from infrared studies that up to pressures of $10 \mathrm{GPa}$ no phase transitions are found due to the close-packed nature of the rigid phase [8]. The intensity $I_{3}$ in the whole range of the rigid phase in nonadecane is pressure independent. It seems to be a general rule (except crossing the minimal void size or changing the phase) that pressure has little influence on the probability of positronium formation.

\section{Gas induced free volume swelling}

Crystal phases expanding in one or more directions under high pressure are now the object of intense studies. To this class belong some biomolecules, but linear negative compressibility was also found in the rotator phase of $n$-alkanes and their derivatives [8]. The PALS method detects the changes of free volume, not the bulk properties of solid, thus the positron studies can supply additional information about this counterintuitive property. The data presented in Sect. 3 indicate that in $n$-nonadecane under pressure the free volumes behave quite normally. With the rise of $p$ the lifetime decreases gently (in both solid phases), thus the free volumes decrease, too. The picture changes radically when the pressure is applied by gaseous atmosphere, in which the samples are submerged.

The sandwich of our samples and positron source was well pressed together and placed directly into the argon filled chamber of the U11 compressor (Unipress, Warsaw). With the rise of pressure the $\tau_{3}$ lifetime increases and at about 


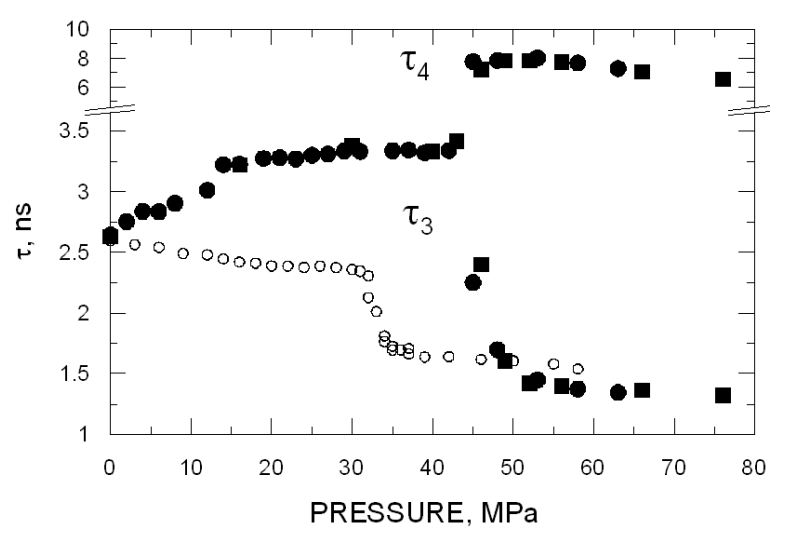

Fig. 5. ortho-Ps lifetime in $n$-nonadecane as a function of pressure for the sample submerged in argon. Full circles - first series; squares - the same sample, second series. Temperature $302 \mathrm{~K}$. Open circles relate to the measurements without gas penetration (Fig. 4). Above the phase transition point the spectra were decomposed into 4 components.

$15 \mathrm{MPa}$ reaches the saturation value $3.3 \mathrm{~ns}$, i.e. is by $0.7 \mathrm{~ns}$ larger than without argon (Fig. 5). Intercalation of argon into the alkane structure introduces large distortions, expanding the intermolecular volumes. The phase transition point to the rigid phase is now shifted upwards to $46 \mathrm{MPa}$ (loading the crystal with argon makes difficult straigthening the kinked molecules, which accompanies the transition to rigid phase). In the rigid phase of argon intercalated nonadecane the long-lived component is found to be far from exponentiality. Splitting it into two components reveals that Ps is located partly in the same gaps between the molecular lamellae as without argon [9], partly in the open spaces allowing Ps to live 7-8 ns. This lifetime value, not seen in nonporous solids, can be explained as a result of microcracks in the samples during phase transformation, positrons now get the direct access to gaseous surrounding. The $o$-Ps lifetime in gaseous argon at these pressures is just the same as observed here.

The sample damage should be irreversible. However, the measurements show that the lifetime spectrum after the reduction of pressure returns to its initial shape. After the full set of measurements up to $70 \mathrm{MPa}$ (the extra-long component has then the intensity of $15 \%$ ) and returning to $43 \mathrm{MPa}$, just $2 \mathrm{MPa}$ below the phase transition point, the 8 ns component has the intensity of $2 \%$ only. This reveals that the nature of spectrum restoration needs further studies.

\section{Variations of Ps intensity with time}

Since late eighties it is known that in polymers and paraffins at low temperature the intensity $I_{3}$ changes with positron irradiation time (usually it rises). The effect was explained by Hirade et al. [10] as the result of the accumulation 
of electrons produced in the ionization track, trapped in defects, and remaining there sufficiently long to take part in Ps formation by subsequent positrons. The effect is negligible at high temperatures (e.g. near the phase transition point in alkanes) due to escape of trapped electrons. Therefore, no $I_{3}$ variation with time elapse is observed in the rotator phase, but it appears after lowering the temperature to the rigid phase. We have tried to observe $I_{3}$ after pressure induced transition to the rigid phase. The result for $n$-heneicosane, $\mathrm{C}_{21} \mathrm{H}_{44}$, is shown in Fig. 6. In this case the intensity diminishes with time; the time constant of that process lies in the range of hours and rises with the increase in pressure [9]. It is clear that the observed effect has nothing common with trapped electrons and results probably from relaxation phenomena. It seemed also interesting to see how rapidly the spectrum parameters change to new values during the phase transition induced by pressure (i.e. in the time scale much shorter than the relaxation mentioned above). In order to observe rapid changes in the spectrum we used the $\mathrm{T}-\mathrm{A}$ converter coupled to the multichannel analyser in the multiscaler mode. The

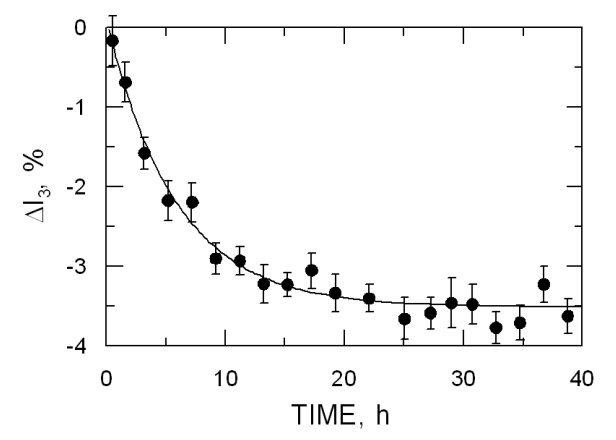

Fig. 6. Decrease in $I_{3}$ intensity (ortho-Ps component) in $n$-heneicosane as a function of storage time after application of pressure $255 \mathrm{MPa}$. Temperature $310 \mathrm{~K}$ [9].

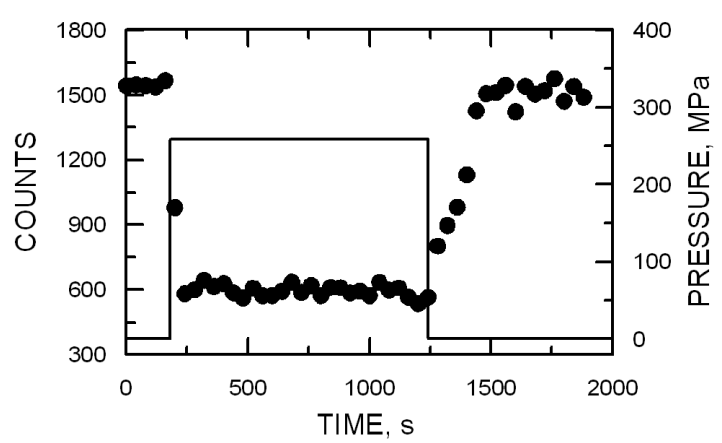

Fig. 7. n-nonadecane. Counting rate in the amplitude window set at the output of time-to-amplitude converter. The window covers the range of delays from $2.15 \mathrm{~ns}$ to $4.0 \mathrm{~ns}$. The pressure as a function of time is shown by solid line. Dwell time (i.e. the time per one channel) is $40 \mathrm{~s}$. 
amplitude window at the output of TAC was set at the tail of distribution, corresponding to large delays (long-lived component). In the rotator phase the number of counts per time unit in such a window is high, in rigid phase - low. The result of measurement with pressure up and down is shown in Fig. 7; the changes occur, as expected, in the negligible time. In the case of samples in argon atmosphere the penetration of gas occurs slowly, thus, similar multiscaler type measurement when the applied pressure is smaller than needed to induce phase transition indicate that the equilibrium value of counting rate is reached after $4 \mathrm{~h}$ of pressure action.

\section{Conclusions}

The pressure studies of $o$-Ps properties in organic solids deserve much more attention than it was in the past. In particular, the counterintuitive modification of Ps properties in high pressure gaseous atmospheres seem to be most interesting.

\section{Acknowledgment}

This work was partly supported by the State Committee for Scientific Research grant No. 5P03B 03121.

\section{References}

[1] J. Kansy, Nucl. Instrum. Methods Phys. Res. A 374, 235 (1996).

[2] T. Goworek, J. Nucl. Radiochem. Sci. 1, 11 (2000).

[3] T. Goworek, T. Suzuki, E. Hamada, K. Kondo, Y. Ito, Chem. Phys. 255, 347 (2000).

[4] T. Goworek, J. Wawryszczuk, R. Zaleski, Chem. Phys. Lett. 387, 433 (2004).

[5] J. Kikas, A. Laisaar, A. Suisalu, A. Kuznetsov, A. Ellervee, Phys. Rev. B 57, 14 (1998).

[6] H. Forsman, P. Andersson, J. Chem. Phys. 80, 2804 (1984).

[7] A.M. Maroncelli, S.P. Qi, H.L. Strauss, R.G. Snyder, J. Am. Chem. Soc. 104, 6237 (1982).

[8] E.B. Sirota, D.M. Singer, H.E. Knight Jr., J. Chem. Phys. 100, 1542 (1994).

[9] T. Goworek, R. Zaleski, J. Wawryszczuk, Chem. Phys. 295, 243 (2003).

[10] C.L. Wang, T. Hirade, F.H.J. Maurer, M. Eldrup, N.J. Pedersen, J. Chem. Phys. 108, 4654 (1998). 